\title{
Research on the overall optimization method of well pattern in water drive reservoirs
}

\author{
Zhibin Zhou $^{1}$ (1) $\cdot$ Jiexiang Wang ${ }^{1} \cdot$ Jing Zhou $^{1}$
}

Received: 20 March 2016/Accepted: 18 June 2016/Published online: 29 June 2016

(c) The Author(s) 2016. This article is published with open access at Springerlink.com

\begin{abstract}
Well pattern optimization is an important part of oil field development design. The heterogeneity and anisotropy of the reservoirs should be considered when the wells are distributed. Considering that well pattern is a complex system with the interaction of each well group, this paper has proposed the overall optimization method of well pattern. In this method, the same of the water breakthrough time is the objective function and the location of each well is optimized. In this paper, the calculation method of the water breakthrough time of heterogeneity/ anisotropy non-piston displacement reservoirs has been derived. Compared to the traditional numerical simulation, the new method is more suitable to solve well arrangement problems because of its higher efficiency. The optimal model has been solved by genetic algorithm. A conceptual model has been established to verify the effectiveness of the proposed method. The optimized method can be used to realize the best match between the well pattern and heterogeneity/anisotropy reservoirs.
\end{abstract}

Keywords Heterogeneous - Anisotropy · Well pattern · Optimization method · Genetic algorithm

\section{Introduction}

Reservoir parameters generally have the heterogeneity and anisotropy (Shah and Mishra 2013; Rose 1982; Yun_hong 2002). For the waterflood development oil field, the water injection well and the corresponding production well

Zhibin Zhou

zhouzhibin_001@163.com

1 China University of Petroleum (East China), Qingdao, China affected by heterogeneous and anisotropic reservoirs are often unable to see the water at the same time, which leads to the imbalance of the displacement process, thus affecting the effect of oil field development. Well pattern design is an important part of oil field development design. If the heterogeneity and anisotropy of the reservoirs can be fully considered when the well is distributed (Liu 2005; Liu and Li 2004), we will make the displacement process more balanced and improve the recovery rate so that we can obtain a higher economic efficiency. Yang et al. (2006) gave the design method of well pattern in anisotropic reservoir in 2006. By adjusting the well pattern and well spacing of the anisotropic reservoirs, the maximum matching with the geological vector was realized and the development effect was improved. However, the well pattern should not only match the anisotropy of the reservoirs, but also match the heterogeneity. Based on the theory of percolation mechanics, reservoir engineering and optimization, a mathematical model for the overall optimization of well pattern in water drive reservoir has been established. Through solving the model, the well pattern matching well with the reservoir is obtained. After that the validity of the method is verified by numerical simulation. Compared to the traditional well pattern optimization, this method has the advantages of fast speed and high efficiency.

\section{Mathematical model for overall optimization of well pattern}

By adjusting the location of wells, well pattern optimization can significantly improve the efficiency of water drive development and obtain higher economic benefits (Beckner and Song 1995). When we optimize overall well pattern 
that different from a single well group, the well group interacted with each other. Decreasing well spacing of a certain well in a well group, it may cause its well spacing increasing in another well group. Therefore, a single well group should not be isolated out in the optimization. The well pattern should be considered as a whole, which is optimized for each well.

\section{Objective function}

Different objective functions can be obtained with different optimization results (Zhang et al. 2010); therefore, the objective function in the establishment of mathematical model for the optimization of well pattern should be first given. Well pattern optimization makes the water breakthrough time of production well tend to be consistent by adjusting the well location in order to maximize the realization of equilibrium displacement. When the water breakthrough time of production well tends to be consistent, the mathematical representation has the least variance. So the minimum variance of the water breakthrough time of each production well has been taken as the objective function of the optimization of the well pattern.

$\min D(t)=\sum_{i=1}^{n}\left[t_{i}-\frac{1}{n} \sum_{i=1}^{n} t_{i}\right]^{2}$

$t_{i}$ - water breakthrough time of each production well, day; $D(t)$-variance of water breakthrough time of each production well; $n-$ number of total production wells.

\section{Constraint condition}

In the process of optimization, the necessary constraints on variables are needed. In the optimization of well pattern, the position of each well can not be beyond the boundary of the oil field and the distance between any two wells can't be too small. Otherwise, it is not economically feasible. Based on the above considerations, the following constraints are shown as:

$$
\begin{aligned}
& \left(x_{i}, y_{i}\right) \in \Omega \quad i=1,2, \ldots, m \\
& d_{i j}=\sqrt{\left(x_{i}-x_{j}\right)^{2}+\left(y_{i}-y_{j}\right)^{2}} \geq d_{\min } \quad i=1,2, \ldots, m ; \\
& \quad j=1,2, \ldots, m
\end{aligned}
$$

$x_{i}, y_{i}, x_{j}, y_{j}-x$ coordinates and $y$ coordinates of the $i$ well and $j$ well, $\mathrm{m} ; \Omega$-reservoir boundary; $d_{i j}$-distance between the $i$ well and $j$ well, $m ; d_{\min }$-minimum well spacing.

To sum up, well pattern optimization problems can be formulated as the location variables satisfy the nonlinear constraints and linear constraints, and the objective function $D(t)$ has been solved to obtain the optimal location of the minimum value $\left(x_{i}^{*}, y_{i}^{*}\right) i=1,2, \ldots, m$.

\section{Non-piston displacement dynamics of heterogeneous and anisotropic reservoirs}

According to the well pattern optimization mathematical model, the water breakthrough time of each production well needs to be calculated during the optimization process. Most of the traditional well location optimization used to be solved by coupling numerical simulation program (Hazlett and Babu 2005; Abukhamsin 2009). The precision of the numerical simulation is high and the adaptability is strong, but the amount of computation is large and the amount of time it takes is long. Because of the complexity of the location optimization problem complexity, it often requires thousands or even more iterations to obtain the optimal solution. Each iteration needs to run the numerical simulation program, which makes the whole process of the optimization process very long, and the efficiency is low. Based on the theory of percolation mechanics and reservoir engineering, the calculation method of water breakthrough time in the heterogeneous and anisotropic reservoirs has been presented to improve the optimization efficiency.

\section{Heterogeneous/anisotropic reservoirs processing}

In the physical property parameters of the reservoir, the permeability has heterogeneity and anisotropy. In the plane two-dimension formation, if the main value of the permeability $k(x, y, 0)$ and $k(x, y, \pi / 2)$ at $x, y$ direction is known, according to the principle of equivalent displacement (Wang et al. 2005), the formula for calculating the permeability $k(x, y, \theta)$ of this point in any direction $\theta$ is shown as:

$k(x, y, \theta)=k(x, y, 0) \cos ^{2} \theta+k(x, y, \pi / 2) \sin ^{2} \theta$

Due to the heterogeneity of permeability, when calculating the water breakthrough time, the average value of permeability between the injection-production wells on the connecting line has been taken.

$\bar{k}=\frac{1}{L} \int_{l} k(x, y, \theta) \mathrm{d} s$

For other reservoir physical parameters, porosity is heterogeneous and scalar. When calculating the time of water breakthrough time, the average value of the connection between the injection wells and the production wells has also been taken. 
$\bar{\phi}=\frac{1}{L} \int_{l} \phi(x, y) \mathrm{d} s$

\section{Calculation of water breakthrough time}

When calculating the water breakthrough time, the oilwater two-phase non-piston displacement was considered, and the capillary force and gravity were ignored. According to the $\mathrm{B}-\mathrm{L}$ displacement theory, the leading edge equation (Wu et al. 2015) is shown as:

$x\left(S_{\mathrm{w}}\right)=\frac{\int_{0}^{t} q_{t} \mathrm{~d} t}{\phi A}\left(\frac{\partial f_{\mathrm{w}}}{\partial S_{\mathrm{w}}}\right)_{S_{\mathrm{w}}}$

$x$-displacement leading edge position, $m ; q_{t}$-injection rate, $m^{3} / d ; A-$ cross-sectional area of reservoir, $m^{2} ; f_{\mathrm{w}}-$ rate of water content; $S_{\mathrm{w}}$-water saturation.

The water breakthrough time of oil well can be obtained by solving the following implicit function:

$d=\frac{\int_{0}^{t_{\mathrm{f}}} q_{t} \mathrm{~d} t}{\phi A}\left(\frac{\partial f_{\mathrm{w}}}{\partial S_{\mathrm{w}}}\right)_{S_{\mathrm{wf}}}$

$D$-well spacing of injection-production wells, $m ; t_{\mathrm{f}}$ water breakthrough time, $d ; S_{\mathrm{wf}}$-water saturation at displacement leading edge. The well spacing $d$, porosity $\varphi$ and cross-sectional area A are the known parameters. According to the phase permeability curve, the relationship between the water content $f_{\mathrm{w}}$ and the water saturation $S_{\mathrm{w}}$ can be obtained. At the same time, the saturation $S_{\mathrm{wf}}$ of the displacement front edge can also be determined.

When injected at constant pressure, the water's injection speed $q_{t}$ is shown as:

$q_{t}=\frac{k A\left(p_{i}-p_{\mathrm{p}}\right)}{\int_{0}^{d} \lambda_{r}^{-1} \mathrm{~d} x}$

$\lambda_{\mathrm{r}}^{-1}=\frac{1}{\lambda_{\mathrm{ro}}+\lambda_{\mathrm{rw}}}$

$p_{i}$-pressure of injection well, Mpa; $p_{\mathrm{p}}$ - pressure of production well, MPa; $\lambda_{\text {ro }}$-relative mobility of the oil phase, $1 / \mathrm{mPa} ; \lambda_{\mathrm{rw}}$-relative mobility of water, $1 / \mathrm{mPa}$.

With the development of the injection water, the water saturation profile is changing continuously (Fig. 1). $\int_{0^{-}}^{\mathrm{d}}$ $a \lambda_{\mathrm{r}}^{-1} \mathrm{~d} x$ also changes at the same time. The displacement front reached $d_{\mathrm{f}}$ at a certain moment before the water breakthrough. The integral term can be written as the sum of two integrals:

$$
\begin{aligned}
\int_{0}^{d} \lambda_{r}^{-1} \mathrm{~d} x= & \int_{0}^{d_{\mathrm{f}}} \frac{1}{\lambda_{\mathrm{ro}}(x)+\lambda_{\mathrm{rw}}(x)} \mathrm{d} x \\
& +\int_{d_{\mathrm{f}}}^{d} \frac{1}{\lambda_{\mathrm{ro}}(x)+\lambda_{\mathrm{rw}}(x)} \mathrm{d} x
\end{aligned}
$$

$0 \leq x<d_{\mathrm{f}}:$

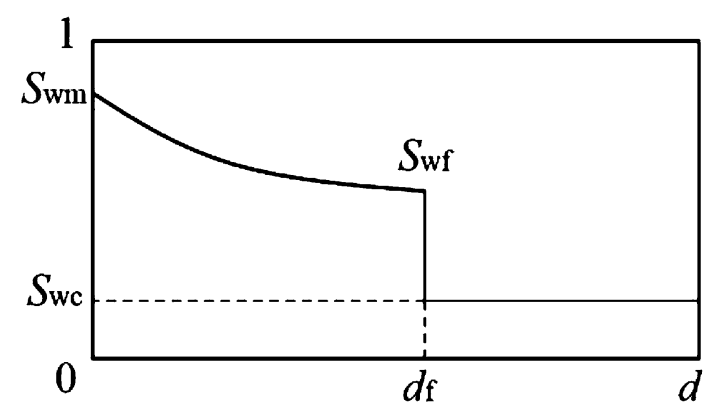

Fig. 1 Non-piston displacement water saturation profile

$\int_{0}^{d_{\mathrm{f}}} \frac{1}{\lambda_{\mathrm{ro}}(x)+\lambda_{\mathrm{rW}}(x)} \mathrm{d} x=\int_{0}^{d_{\mathrm{f}}} \frac{1}{k_{\mathrm{ro}}(x) / \mu_{\mathrm{o}}+k_{\mathrm{rw}}(x) / \mu_{\mathrm{w}}} \mathrm{d} x$

$d_{\mathrm{f}} \leq x \leq d$, the water saturation $S_{\mathrm{wc}}$ is constant:

$\int_{d_{\mathrm{f}}}^{d} \frac{1}{\lambda_{\mathrm{ro}}(x)+\lambda_{\mathrm{rw}}(x)} \mathrm{d} x=\frac{\left(d-d_{f}\right)}{k_{\mathrm{ro}}\left(S_{\mathrm{wc}}\right) / \mu_{\mathrm{o}}+k_{\mathrm{rw}}\left(S_{\mathrm{wc}}\right) / \mu_{\mathrm{w}}}$

$k_{\mathrm{ro}}$-relative permeability of oil; $k_{\mathrm{rw}}$-relative permeability of water; $\mu_{\mathrm{o}}$-viscosity of oil phase, Mpa; $\mu_{\mathrm{w}}$-viscosity of water phase, Mpa.

There is no direct function relationship between the relative permeability $k_{\mathrm{ro}}, k_{\mathrm{rw}}$ and the position $x$, but it can be established by means of formula (7). It is difficult to get a clear expression of the function. Therefore, the numerical method is generally used to calculate the first integral. Taking into account the actual data provided by the relative permeability curves tend to be scattered points, the first integral is solved by using the subsection trapezoid formula.

By formula (7) and formula (9), the time of injection speed needs be known in order to obtain the water breakthrough time. The injection speed is related to the position of the displacement leading edge at any time, and the displacement leading edge position is a function of the total injection quantity. Therefore, the iterative method should be used in the calculation of water breakthrough time. The calculation steps are shown as:

1. Assuming the initial time $t=0$ and initial displacement leading edge position $d_{\mathrm{f}}=0$, the phase permeability curve $\left[S_{\mathrm{w} 1}, S_{\mathrm{w} 2}, \ldots, S_{\mathrm{w} n}\right],\left[k_{\mathrm{ro} 1}, k_{\mathrm{ro} 2}, \ldots, k_{\mathrm{ro} n}\right]$, $\left[k_{\mathrm{rw} 1}, k_{\mathrm{rw} 2}, \ldots, k_{\mathrm{rw} n}\right]$ are given. The water cut sequence $\left[f_{\mathrm{w} 1}, f_{\mathrm{w} 2}, \ldots, f_{\mathrm{w} n}\right]$ has been calculated. The forward difference has been used to calculate the derivative sequence of water content to saturation.

$\left.\frac{\partial f_{\mathrm{w}}}{\partial S_{\mathrm{w}}}\right|_{S_{\mathrm{w} i}}= \begin{cases}\frac{f_{\mathrm{w} i+1}-f_{\mathrm{w} i}}{S_{\mathrm{w} i+1}-S_{\mathrm{w} i}} & i=1,2, \ldots, n-1 \\ 0 & i=n\end{cases}$

2. According to formula (7), the corresponding saturation position sequence $\left[d_{1}, d_{2}, \ldots, d_{n}\right]$ has been calculated. 
3. According to formula (11), the integral term $\int_{0}^{d} \lambda_{\mathrm{r}}^{-1} \mathrm{~d} x$ has been calculated. Among them, the first segment is calculated by subsection trapezoid formula.

$$
\begin{aligned}
& \int_{0}^{d_{\mathrm{f}}} \frac{1}{\lambda_{\mathrm{ro}}(x)+\lambda_{\mathrm{rw}}(x)} \mathrm{d} x \\
& =\sum_{i=1}^{n-1}\left\{\frac{d_{i}-d_{i+1}}{2}\left[\frac{1}{\frac{k_{\mathrm{ro}}\left(S_{\mathrm{w} i}\right)}{\mu_{\mathrm{o}}}+\frac{k_{\mathrm{rw}}\left(S_{\mathrm{w} i}\right)}{\mu_{\mathrm{w}}}}+\frac{1}{\frac{k_{\mathrm{ro}}\left(S_{\mathrm{w} i}\right)}{\mu_{\mathrm{o}}}+\frac{k_{\mathrm{rw}}\left(S_{\mathrm{w} i+1}\right)}{\mu_{\mathrm{w}}}}\right]\right\}
\end{aligned}
$$

4. According to formula (9), the injection rate $q_{t}$ and the cumulative injection $W_{i}=W_{i}+q_{t} \Delta t$ have been calculated at that time.

5. The displacement leading edge position $d_{\mathrm{f}}$ has been calculated.

6. If the displacement front position $d_{\mathrm{f}}<$ the injection production well spacing $d$, let $t=t+\Delta t$ and go to step (2). Otherwise, the iteration ends.

\section{Solution of mathematical model}

Well location optimization problem is an optimization problem of large-scale and multipeak value with constraint. At present, the optimization algorithms are mainly divided into two categories (Onwunalu 2010; Emerick et al. 2009; Guan et al. 2005): random search algorithm and gradient algorithm. The random search algorithm has a large scale of computing, and it can avoid the local optimal solution by controlling mechanism or algorithm operation. The gradient algorithm is efficient, but sometimes it can fall into the local optimal solution. Combined with the characteristics of the mathematical model of well pattern optimization, this paper chooses the genetic algorithm as the solution algorithm of this problem.

The genetic algorithm is a heuristic random search method based on biological evolution. The algorithm does not require the form of function. The algorithm starts from the initial population and has implicit parallelism, so it has a better search efficiency and has better global searching ability. The basic steps of using genetic algorithm to solve the well pattern optimization model are as follows:

1. The initial population is generated, the coding strategy is selected, and the parameter set is converted to a bit string space.

2. Three basic operators (selection, crossover and mutation) are used to form the population.

3. Determine whether the population performance can meet the objective function or have completed the number of iterations. If the condition is satisfied, the result is output. Otherwise, the population is used as the parent population, and the iteration is carried out again.

\section{Sample analysis}

Two-dimensional two-phase reservoir model of fluvial facies is established. The grid is $100 \times 100 \times 1$, and the size of the grid is $\Delta x=\Delta y=\Delta z=10 \mathrm{~m}$. The permeability distribution is shown in Fig. 2. The porosity is 0.3 , the irreducible water saturation is 0.3 , and the residual oil saturation is 0.3 .

Then the anti-nine-spot well pattern has been arranged. There are 25 wells, including 21 production wells and 4 water injection wells. The bottom hole pressure of the water injection well is $23 \mathrm{MPa}$, and the bottom hole pressure of the production well is $18 \mathrm{MPa}$. Before and after optimization, the well pattern arrangement is shown in Figs. 3 and 4. Among them, WELL7, WELL9, WELL17 and WELL19 are the water injection wells, and the other wells are the production wells.

As we can see, the well pattern is no longer a rule after the optimization. Because the irregular distribution of well pattern is matching with the permeability distribution of reservoirs, it makes the water fingering phenomenon reduce. A water injection well corresponds to the eight production wells around in every well group, that is, there are eight corresponding relations between the injection and production wells. The connection numbers of the WELL7 and the surrounding production wells (WELL1, WELL2, WELL3, WELL6, WELL8, WELL11, WELL12, WELL13) have been defined as $1,2, \ldots, 8$. By analogy, the

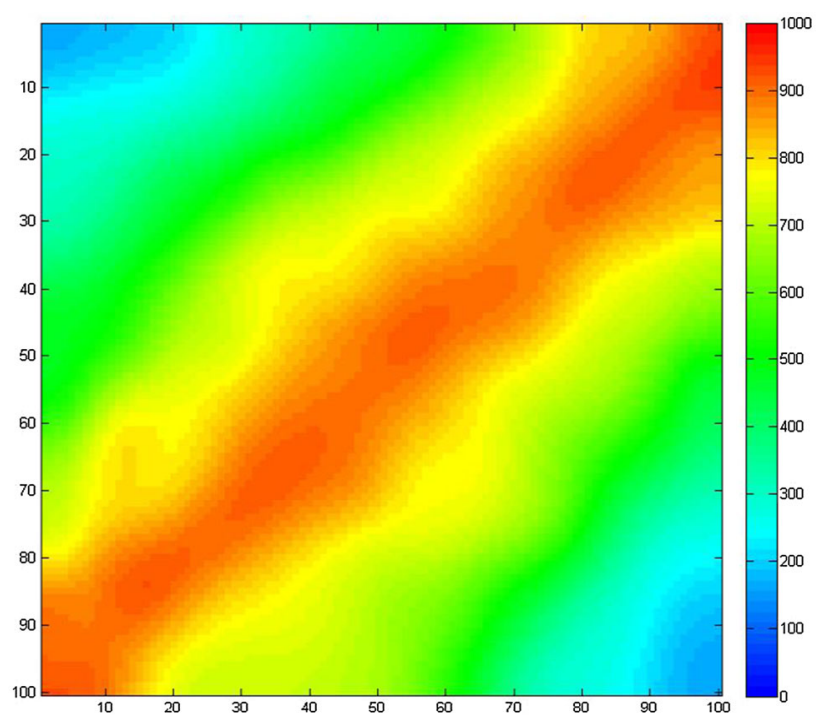

Fig. 2 Permeability distribution 


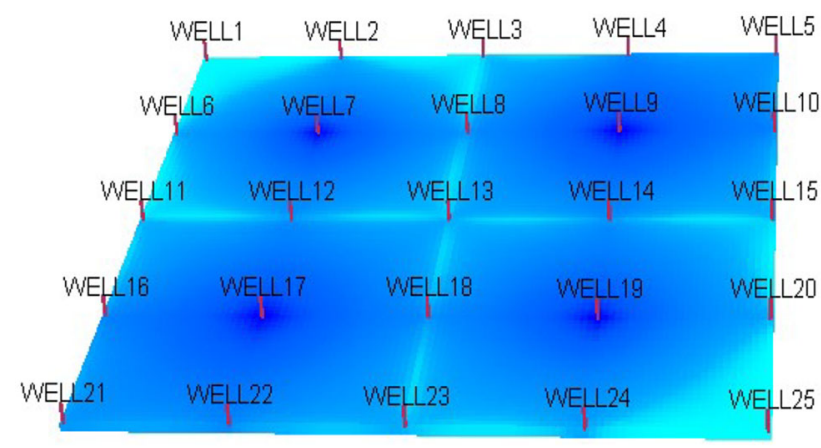

Fig. 3 Well location before optimization

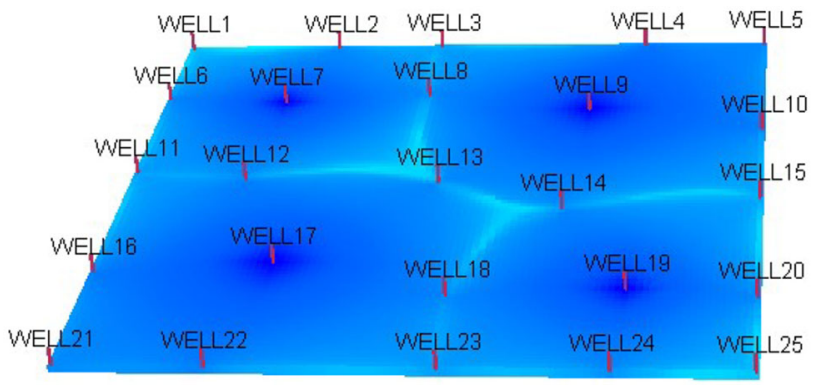

Fig. 4 Well location after optimization

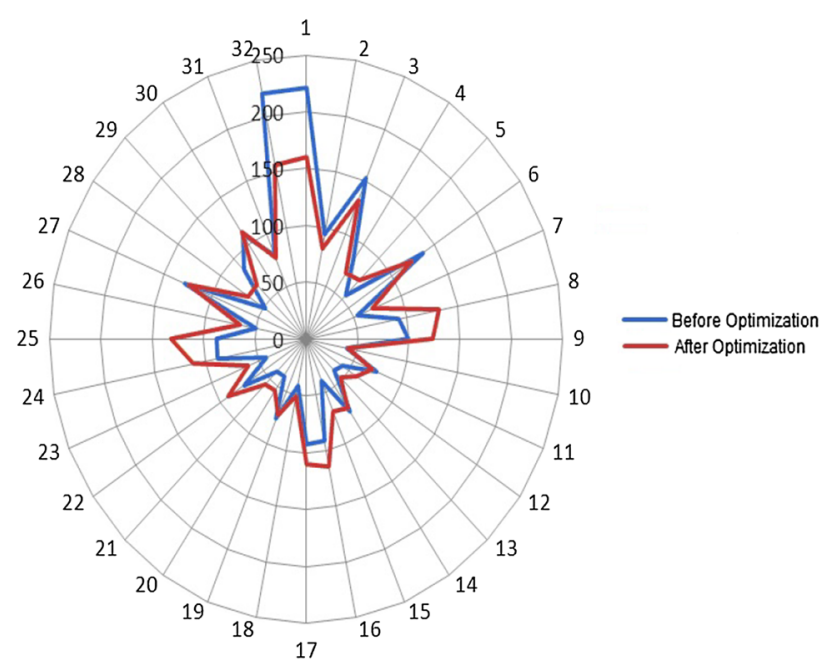

Fig. 5 Comparison of water breakthrough time before and after optimization

connection numbers of the water injection well (WELL17, WELL9, WELL19) and their surrounding production wells have been defined. There are a total of 32 injection-production lines. The water breakthrough time of each injection-production line is shown in Fig. 5. The water breakthrough time of each well is more consistent after optimization.

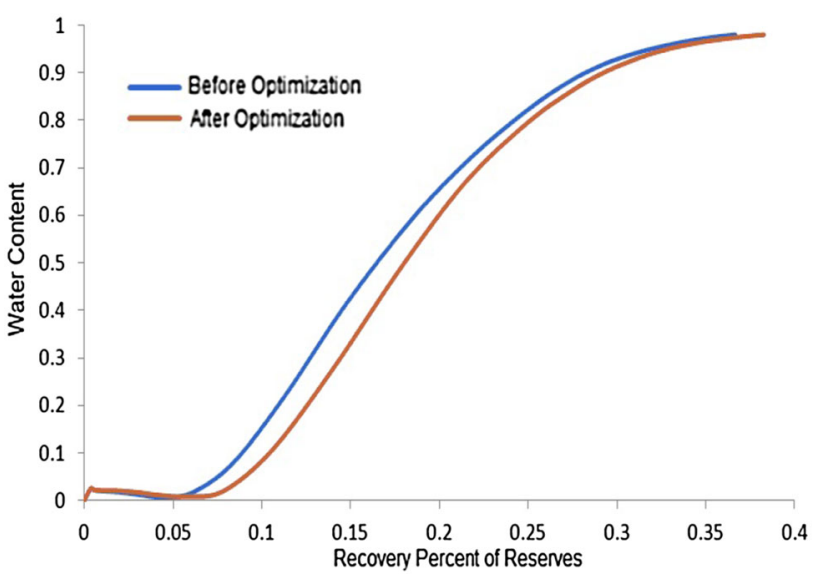

Fig. 6 Comparison of the recovery degree and water content curve

The well location of before and after optimization has been input to numerical simulation software for numerical simulation calculation. The results can be seen from the recovery degree and water content curve (Fig. 6). After using the optimized well pattern, the water content is lower and the final recovery degree is higher.

\section{Conclusions}

1. A mathematical model of the overall optimization of well pattern has been established. The target function is to keep the water breakthrough time consistent in each production well, and the adjustment is carried out for each well in the well pattern in order to realize the maximum equilibrium displacement.

2. The calculation method of the water breakthrough time of heterogeneity/anisotropy non-piston displacement reservoirs has been derived. Compared to the traditional numerical simulation, the new method is more suitable for the large-scale and multivariable well pattern optimization problems. This method has higher speed and efficiency.

3. A conceptual model has been established to verify the effectiveness of the proposed method. The results indicated that the optimized method can be used to realize the best match between the well pattern and heterogeneity/anisotropy reservoirs. After using the optimized well pattern, the water content is lower and the final recovery degree is higher. Therefore, the overall optimization method is feasible and effective.

Acknowledgments The study was supported by the National 863 Program of China (2009AA063402). 
Open Access This article is distributed under the terms of the Creative Commons Attribution 4.0 International License (http:// creativecommons.org/licenses/by/4.0/), which permits unrestricted use, distribution, and reproduction in any medium, provided you give appropriate credit to the original author(s) and the source, provide a link to the Creative Commons license, and indicate if changes were made.

\section{References}

Abukhamsin AY (2009) Optimization of well design and location in a real field. Stanford University

Beckner BL, Song X (1995) Field development planning using simulated annealing-optimal economic well scheduling and placement. In: SPE annual technical conference and exhibition. Society of Petroleum Engineers

Emerick AA, Silva E, Messer B et al (2009) Well placement optimization using a genetic algorithm with nonlinear constraints. In: SPE reservoir simulation symposium. Society of Petroleum Engineers

Guan X, Wei L, Yang J (2005) Optimization of operation plan for water injection system in oilfield using hybrid genetic algorithm. Acta Pet Sin 26(3):114

Hazlett RD, Babu DK (2005) Optimal well placement in heterogeneous reservoirs via semi-analytic modeling. SPE J 10(03):286-296
Liu YT (2005) Well location in water-flooding anisotropic oil reservoirs. Shiyou Kantan Yu Kaifa (Pet Explor Dev) 32(5):101-104

Liu D, Li S (2004) Concept of vector well pattern and method of well pattern arrangement. J Jianghan Pet Inst 4:039

Onwunalu JE (2010) Optimization of field development using particle swarm optimization and new well pattern descriptions. Stanford University

Rose W (1982) Permeability anisotropy in low permeability formations. In: SPE unconventional gas recovery symposium. Society of Petroleum Engineers

Shah N, Mishra P (2013) Oil production optimization: a mathematical model. J Pet Explor Prod Technol 3(1):37-42

Wang D, Zhou Y, Ma P et al (2005) Vector properties and calculation model for directional rock permeability. Rock Soil Mech Wuhan 26(8): 1294

Wu K, Li X, Ruan M et al (2015) Dynamic tracking model for the reservoir water flooding of a separated layer water injection based on a well temperature log. J Pet Explor Prod Technol $5(1): 35-43$

Yang L, Duan-ping W, Chuan-Liang L (2006) Vectorial well arrangement in anisotropic reservoirs. Pet Explor Dev 33(2):225-227

Yun_hong D (2002) The development well-pattern of low and anisotropic permeability reservoirs. Acta Pet Sin 2:019

Zhang K, Li Y, Yao J et al (2010) Theoretical research on production optimization of oil reservoirs. Acta Pet Sin 31(1):78-83 